Letícia Krauss Silva 1

Tomaz Pinheiro da Costa 2

Aldo Franklin Reis 2

Neiw Oliveira Iamada 2

Andréa Paula de Azevedo 2

Carla Pontes de Albuquerque 1

\section{Avaliação da qualidade da assistência hospitalar obstétrica: uso de corticóides no trabalho de parto prematuro}

\author{
Assessment of quality of obstetric care \\ and corticoid use in preterm labor
}

1 Centro de Avaliação de Programas, Serviços e Tecnologias de Saúde, Escola Nacional de Saúde Pública, Fundação Oswaldo Cruz. Rua Leopoldo Bulhões 1480 , sala 708, Rio de Janeiro, $R J$ 21045-900, Brasil.

2 Departamento de Ginecologia e Obstetrícia, Faculdade de Medicina, Universidade Federal do Rio de Janeiro. Av. Brigadeiro Trompouski s/no, Bloco K, Centro de Ciências da Saúde, Cidade Universitária, Ilha do Fundão, Rio de Janeiro, RJ 21941-590, Brasil.

\begin{abstract}
This study aimed to assess quality of obstetric care for preterm labor patients, using referents, indicators, and standards derived from scientific evidence, focusing on antenatal corticotherapy. Available metanalyses and randomized controlled trials were examined to establish referents, defining indicators and estimating process and outcome standards for the present study. Data from hospital discharge summaries of seven public maternity hospitals in Rio de Janeiro were analyzed. The standard of process used was 100\%. It was not possible to estimate outcome standards, since the necessary adjustment for gestational age was not feasible. Utilization of antenatal corticotherapy in the present study was very low, about $4 \%$ and $2 \%$, considering patients up to 33 weeks and 6 days and 36 weeks and 6 days, respectively. Failure to use antenatal corticotherapy when formally indicated deserves attention by health planners and managers, considering: a) the ease in incorporating such a technology, in contrast to the adequate incorporation of special/intensive neonatal care; b) benefits and costs associated with this technology compared to those of delivering neonatal care to premature babies.
\end{abstract}

Key words Prematurity; Birth; Maternal and Child Health

Resumo O objetivo do estudo foi avaliar a qualidade da assistência ao trabalho de parto prematuro, utilizando referentes, indicadores e padrões derivados de evidências científicas - ensaios clínicos controlados e meta-análises -, tomando como caso a corticoterapia anteparto. Foram analisados dados de sumários de alta relativos a sete maternidades públicas do Rio de Janeiro. O padrão utilizado para a análise de processo foi de 100\%. Não foi possível estimar padrões de resultado - incidência esperada de síndrome de angústia respiratória e mortalidade neonatal - para os referentes previstos, em razão da impossibilidade de ajustar os resultados para idade gestacional, poderoso fator interveniente. A utilização da corticoterapia antenatal pelos serviços analisados foi irrisória, cerca de $4 \%$ e $2 \%$, para os referentes relativos a pacientes com menos de 34 semanas e com até 36 semanas de idade gestacional, respectivamente. A falha no uso da corticoterapia antenatal quando indicada merece a atenção de planejadores e gestores do setor, tendo em vista a fácil incorporação de tal tecnologia, bem como os benefícios e os custos desta, em comparação com aqueles associados à assistência neonatal a bebês prematuros.

Palavras-chave Prematuridade; Parto; Saúde Materno-Infantil 


\section{Introdução}

A avaliação da qualidade da assistência hospitalar visa dimensionar a adequação da utilização de procedimentos eficazes e relativamente seguros e baratos, assim como os resultados obtidos (efetividade) por seu uso.

O desenvolvimento acelerado de tecnologias e a incorporação destas têm implicado altos custos, o que levou os países desenvolvidos, especialmente a partir de meados da década de 80 , a uma política de verificação dos benefícios reais proporcionados pela aplicação destas (Roper et al., 1988; GAO/USA, 1992; Banta \& Luce, 1993; OTA/USA, 1994). No que diz respeito à realidade da assistência perinatal no Brasil, estudos anteriores mostraram uma relação extremamente desfavorável entre efetividade e custos de serviços neonatais no Rio de Janeiro (Krauss-Silva, 1992). Esses achados indicam a necessidade de investimento na qualidade da assistência neonatal e valorizam a integração desta com a clínica obstétrica, de forma a proporcionar melhores condições de viabilidade fetal.

Por outro lado, a utilização de tecnologias obstétricas de forma adequada tem como conseqüências um melhor resultado perinatal e a racionalização de recursos, resultando em menores custos assistenciais. Especialmente com relação ao parto prematuro, alguns procedimentos obstétricos, relativamente simples, previnem complicações neonatais que traduzem-se por aumento de mortalidade e morbidade (inclusive seqüelas), poupando ainda a utilização de tecnologias terciárias de alto custo (assistência respiratória, longa internação em unidade de tratamento intensivo neonatal, dentre outros). Mais recentemente, a literatura mostra que o uso profilático e/ou terapêutico de surfactantes exógenos (aplicação endotraqueal) em prematuros com alto risco de desenvolver síndrome de angústia respiratória (SAR) e outras complicações pulmonares tem apresentado resultados promissores: reduz a incidência de pneumotórax e a mortalidade neonatal, além de aumentar a sobrevivência sem displasia bronco-pulmonar, sendo que nenhum efeito adverso do tratamento profilático foi identificado (Soll, 1997). Este procedimento, todavia, requer considerável expertise, além de que precisa ser complementado por assistência ventilatória, embora torne o uso desta menos prolongado (Soll \& Morley, 1997). Assim, o custo total da assistência neonatal a prematuros permanece muito elevado em média quando comparado aos custos dos procedimentos obstétricos que diminuem a fre- qüência ou a duração do uso de tecnologias neonatais.

A prematuridade - nascimento antes de 37 semanas completas de gestação - representa de $6 \%$ a $10 \%$ das gestações, sendo responsável por cerca de $70 \%$ de todas as mortes perinatais (Lockwood, 1994). No Rio de Janeiro, a incidência de prematuridade em maternidades públicas é de aproximadamente $12 \%$, representando cerca de $85 \%$ dos óbitos neonatais, excluídas as malformações congênitas letais (SES/RJ, 1990). Apesar de muitos partos prematuros estarem associados a outras patologias e afecções gestacionais (gestação múltipla, hemorragia acidental, hipertensão, dentre outros), 50\% deles têm causa idiopática (Chalmers et al., 1993), o que limita a prevenção. Nos casos ditos idiopáticos, é a qualidade da assistência hospitalar que permitirá uma intervenção adequada para que se melhore o resultado perinatal.

No caso de trabalho de parto prematuro, retiradas as contra-indicações clínicas, o uso de tocolíticos está indicado (Crowley, 1996) para a reversão do trabalho de parto, conjuntamente à corticoterapia pré-parto (NIH/USA, 1994; Crowley, 1996), para acelerar a maturação fetal, diminuindo a ocorrência de síndrome de angústia respiratória, hemorragia intraventricular cerebral e morte neonatal.

Cerca de $25 \%$ dos partos prematuros são eletivos, variando esse índice de acordo com a disponibilidade de tecnologia na assistência obstétrica e neonatal (Chalmers et al., 1993). Nesses casos (crescimento intra-uterino retardado e patologias maternas graves, dentre outros), o planejamento da interrupção gestacional pode ser auxiliado pela realização de amniocentese para testar a maturidade fetal e, em caso de imaturidade, a administração de corticóide antes do parto também está indicada.

\section{Objetivo}

O objetivo do presente trabalho foi o de avaliar a qualidade da assistência obstétrica no que se refere à utilização (processo) e ao resultado da corticoterapia anteparto no trabalho parto prematuro em maternidades públicas do Rio de Janeiro.

\section{Metodologia}

Foram observadas as seguintes etapas metodológicas: revisão geral da literatura pertinente ao estudo; revisão sistemática (Bailey, 1987; Sackes et al., 1987; Yusuf et al., 1991; Oxman \& 
Guyatt, 1992; Oxman et al., 1994; Schulz et al., 1995; Chalmers \& Altman, 1995) da literatura relativa a evidências da eficácia/segurança da corticoterapia antenatal, particularmente aquelas derivadas de ensaios clínicos randomizados; análise dos dados disponíveis para a avaliação dos serviços; elaboração de referentes, indicadores e padrões - com base na análise dos ensaios mencionados e nos dados de boa qualidade disponíveis para o presente estudo a serem utilizados nesta avaliação.

Revisão sistemática dos estudos sobre efeitos da corticoterapia antenatal e análise de estudos correlatos

A revisão relativa aos efeitos da corticoterapia antenatal, em especial para pacientes em trabalho de parto prematuro, foi baseada no trabalho de Crowley (1996). A autora selecionou, dentre algumas dezenas de ensaios existentes, estudos controlados randomizados, bem delineados e com o viés reduzido pelo uso de injeções de placebo e alocação para o tratamento cega (Liggins \& Howie, 1972; Block et al., 1977; Morrison et al., 1978; Papageorgiou et al., 1979; Taeusch Jr. et al., 1979; Doran et al., 1980; Schutte et al., 1980; Teramo et al., 1980; US Antenatal Steroid Trial, 1981; Schmidt et al., 1984; Morales et al., 1986; Gamsu et al., 1989; Parsons et al., 1988; Cararach et al., 1990; Crowley et al., 1990; Carlan et al., 1991; Garite et al., 1992; Kari et al., 1994; Silver et al., 1995).

\section{a) População estudada e esquemas terapêuticos}

Houve variação na delimitação da população dos ensaios, incluindo-se, via de regra, o trabalho de parto prematuro idiopático, segundo critérios geralmente não definidos, e excluindo-se o trabalho de parto prematuro associado a variadas condições (geralmente contra-indicações à tocólise), estas, relativamente pouco prevalentes.

Outra falta de homogeneidade na população dos estudos foi quanto à faixa de idade gestacional: foram feitos diferentes recortes no intervalo entre 25 e 37 semanas, embora grande parte dos estudos tenha compreendido a faixa entre 28 e 34 semanas. Além disso, os ensaios utilizaram em geral três métodos para esse recorte: avaliação pela data da última menstruação (DUM)/ultra-sonografia (USG) (quatro ensaios), avaliação pela DUM/USG complementada pelo critério lecitina/esfingomielina (LE) maior que 2 (aproximadamente) (seis ensaios) e avaliação pela DUM/USG complementada pela avaliação pós-natal (critério idade gestacional segundo Dubowitz maior que 34 semanas) (dois ensaios).

Quanto aos esquemas terapêuticos testados - $24 \mathrm{mg}$ de betametasona ou de dexametasona ou $2 \mathrm{~g}$ de hidrocortisona - apenas para os dois primeiros existe evidência suficiente de eficácia, com percentuais semelhantes. O da betametasona foi o mais utilizado. Essas drogas estavam disponíveis nos serviços a serem avaliados.

\section{b) Medidas de resultado}

Quase todos os ensaios mediram a incidência da síndrome de angústia respiratória (SAR) no recém-nascido de acordo com a intenção de tratar e com o "tempo de tratamento" - tempo decorrido entre o início do tratamento e o parto. Alguns ensaios mediram aquela incidência segundo a idade gestacional à admissão, outros ao parto e outros ainda segundo a idade gestacional do recém-nascido. Alguns trabalhos mediram a freqüência de outras morbidades associadas ao nascimento prematuro como hemorragia intraventricular e enterocolite necrotizante. Muitos trabalhos mediram a freqüência de morte neonatal e alguns a de morte fetal.

\section{c) Eficácia}

A razão de taxas (RT) para SAR do grupo tratado sobre o grupo controle do conjunto dos ensaios bem delineados sobre corticoterapia antenatal foi de 0,64 com um intervalo de confiança de 0,57 a 0,75 (intenção de tratar), sendo, portanto, a eficácia geral dos estudos de 0,36 , isto é, de $36 \%$ (Tabela 1 ). Com relação à morte neonatal, a RT foi de 0,37 , com intervalo de confiança (95\%) de 0,51 a 0,77 (Tabela 2) e a eficácia foi de $63 \%$. As razões de taxas para hemorragia intraventricular e enterocolite necrotizante foram 0,30 (IC (95\%) 0,14 a 0,66) e 0,60 (IC (95\%) 0,33 a 1,09), respectivamente, indicando benefício para a prevenção da hemorragia intraventricular e forte tendência ao benefício para a enterocolite necrotizante. Os fatores intervenientes mais relevantes no resultado da corticoterapia foram a idade gestacional e a completude de tratamento.

Os resultados segundo idade gestacional ao parto dos ensaios clínicos nos mostram um claro benefício na prevenção da SAR nas gestações com menos de 34 semanas $(\mathrm{RT}=0,47$ e $0,37<$ $\mathrm{RT}<0,58)$, com menos de 32 semanas ( $\mathrm{RT}=0,46$ e $0,34<\mathrm{RT}<0,63$ ) e um benefício menor na faixa de menos de 30 semanas ( $\mathrm{RT}=0,77$ e $0,65<\mathrm{RT}<$ $0,91)$. Nos subgrupos com nascimentos acima de 34 semanas $(\mathrm{RT}=0,66$ e $0,34<\mathrm{RT}<1,27)$ e com 
Incidência de síndrome da angústia respiratória do recém-nascido (SAR) de uma maneira geral (intenção de tratar) em ensaios clínicos de corticoterapia antenatal versus placebo.

\begin{tabular}{|c|c|c|c|c|}
\hline Trabalhos & № eventos & Total tratados & № eventos & Total do controle \\
\hline Liggins \& Howie, 1972 & 49 & 532 & 84 & 538 \\
\hline Block et al., 1977 & 5 & 69 & 12 & 61 \\
\hline Schutte et al., 1980 & 11 & 64 & 17 & 58 \\
\hline Taeusch Jr. et al., 1979 & 7 & 56 & 14 & 71 \\
\hline Doran et al., 1980 & 4 & 81 & 10 & 63 \\
\hline Teramo et al., 1980 & 3 & 38 & 3 & 42 \\
\hline Gamsu et al., 1989 & 7 & 131 & 16 & 137 \\
\hline $\begin{array}{l}\text { Collaborative Group on Antenatal } \\
\text { Steroid Therapy, } 1981\end{array}$ & 42 & 371 & 59 & 372 \\
\hline Morales, 1986 & 30 & 121 & 63 & 124 \\
\hline Papageorgiou et al., 1979 & 7 & 71 & 23 & 75 \\
\hline Kari et al., 1994 & 35 & 95 & 45 & 94 \\
\hline Cararach et al., 1990 & 1 & 12 & 0 & 6 \\
\hline Garite et al., 1992 & 21 & 40 & 28 & 42 \\
\hline Carlan et al., 1991 & 1 & 11 & 4 & 13 \\
\hline Morrison et al., 1978 & 6 & 67 & 14 & 59 \\
\hline Parsons et al., 1988 & 3 & 23 & 3 & 22 \\
\hline Schmidt et al., 1984 & 17 & 49 & 10 & 31 \\
\hline Silver et al., 1995 & 43 & 54 & 34 & 42 \\
\hline Total & 292 & 1885 & 439 & 1850 \\
\hline
\end{tabular}

Razão das taxas obtida $=0,64$ (I.C. $95 \%=0,57-0,75)$.

Obs.: Tabela adaptada da revisão de Crowley (1996 - Oxford Database).

Tabela 2

Incidência de morte neonatal em ensaios clínicos de corticoterapia antenatal versus placebo (intenção de tratar).

\begin{tabular}{lrrrr}
\hline Trabalhos & No eventos & Total tratados & № eventos & Total do controle \\
\hline Liggins \& Howie, 1972 & 36 & 532 & 60 & 538 \\
Block et al., 1977 & 1 & 69 & 5 & 61 \\
Schutte et al., 1980 & 3 & 64 & 12 & 58 \\
Taeusch Jr. et al., 1979 & 8 & 56 & 10 & 71 \\
Doran et al., 1980 & 4 & 81 & 11 & 63 \\
Collaborative Group on Antenatal & 32 & 371 & 34 & 372 \\
Steroid Therapy, 1981 & & & & 42 \\
Garite et al., 1992 & 9 & 121 & 11 & 124 \\
Morales et al., 1986 & 7 & 95 & 13 & 94 \\
Kari et al., 1994 & 6 & 131 & 9 & 137 \\
Gamsu et al., 1989 & 14 & 23 & 1 & 22 \\
Parsons et al., 1988 & 0 & 49 & 4 & 31 \\
Schmidt et al., 1984 & 5 & 67 & 7 & 59 \\
Morrison et al., 1978 & 3 & 71 & 5 & 75 \\
Papageorgiou, 1979 & 1 & 204 & 1747 \\
Total & 129 & & & \\
\hline
\end{tabular}

Razão das taxas obtida $=0,63$ (I.C. $95 \%=0,51-0,77)$.

Obs.: Tabela adaptada da revisão de Crowley (1996 - Oxford Database). 
menos de 28 semanas $(\mathrm{RT}=0,71$ e $0,37<\mathrm{RT}<$ 1,35), embora haja tendência ao benefício, os resultados quanto à prevenção da SAR não são estatisticamente significativos. Pode-se concluir que a faixa de idade gestacional de maior eficácia é a faixa entre 30 e 34 semanas.

Quanto ao tempo de tratamento, ficou evidenciada a eficácia na prevenção da SAR apenas para a exposição sem intervalo de tempo definido entre administração do corticóide e nascimento (intenção de tratar, Tabela 1 (RT: $0,64$ e $0,57<\mathrm{RT}<0,75)$ e para o intervalo de tempo entre 24 h e 7 dias, com eficácia maior (RT: $0,46$ : e $0,33<\mathrm{RT}<0,64)$. A análise dos resultados para o tempo de exposição menor que $24 \mathrm{~h}$ mostra apenas tendência de benefício, uma vez que o intervalo de confiança corrrespondente à razão de taxas inclui o valor 1 .

Considerando-se o objetivo do presente estudo de analisar a qualidade da assistência relacionada ao problema do trabalho de parto prematuro, e não a de todo o possível referente da tecnologia corticoterápica antenatal, isto é, trabalho de parto prematuro e interrupção eletiva da gestação (parto prematuro planejado por patologias feto-maternas), procurou-se avaliar a eficácia da corticoterapia para o subgrupo trabalho de parto prematuro e delimitar as exclusões a serem consideradas para esse trabalho.

Nenhum dos ensaios selecionados incluiu exclusivamente pacientes gestantes em trabalho de parto prematuro. A razão de taxas correspondente aos ensaios com pelo menos $85 \%$ das pacientes em trabalho de parto prematuro (Papageorgiou et al., 1979; Doran et al., 1980; Gamsu et al., 1989) foi de 0,36 (com intervalo de confiança (95\%) de 0,24 a 0,54 ) para o resultado incidência de SAR, correspondendo a uma eficácia (intenção de tratar) 64\%, maior que aquela do conjunto dos ensaios. A estimativa da eficácia na diminuição de morte neonatal em pacientes em trabalho de parto prematuro não foi possível pela baixa freqüência desse evento nos estudos citados.

Os eventos excluídos foram classificados segundo sua natureza em: a) "exclusões absolutas”, relacionadas diretamente ao emprego de corticóides e/ou condição obstétrica, por impor risco de vida à mãe, ao bebê ou a ambos, e aos casos de morte fetal (antes da admissão); b) “exclusões relativas", situações clínicas que podem tornar impertinente o emprego da corticoterapia antenatal.

\section{d) Segurança}

Os estudos que investigaram o uso antenatal de corticóides e o risco de infecção perinatal, concluíram que não há risco aumentado de infecção perinatal (RT $=0,84$, com intervalo de confiaça (95\%) de 0,60 a 1,19). Alguns estudos efetuaram o acompanhamento dos bebês submetidos à corticoterapia antenatal e não evidenciaram comprometimento neurológico ou intelectual (Chalmers et al., 1993; Crowley, 1996).

\section{Fonte e qualidade dos dados dos serviços}

O banco de dados utilizado para esta investigação foi o de sumários de alta da História Clínica Perinatal (HCP) - sistema de informação adaptado do original do Centro Latinoamericano de Perinatologia da Organização Panamericana da Saúde, principalmente, para possibilitar as análises de processo e da relação processo-resultado (Krauss-Silva,1996), dentro de um programa de avaliação da qualidade. O sistema de informação utilizado incluía programa de entrada de dados. Os dados disponíveis foram aferidos - e corrigidos quando necessário - de forma a avaliar o preenchimento dos mesmos e a qualidade das informações existentes. Para isso foi feita a análise das freqüências simples das variáveis relevantes para o estudo e a análise de sua distribuição, segundo variáveis selecionadas. Os indicadores e padrões adiante apresentados foram aplicados na avaliação da qualidade da assistência a cerca de 20.000 partos ocorridos em sete maternidades públicas, não universitárias, de níveis secundário (quatro) e terciário (três), da Cidade do Rio de Janeiro.

O sumário materno dispunha de informação relacionada a diagnósticos materno-fetais e sobre idade gestacional (DUM e USG). O presente estudo, para fins de cálculo da idade gestacional, utilizou a USG (independente do período gestacional da realização da mesma), sempre que informações sobre esta estivessem disponíveis, ficando a DUM como default (Anderson et al.,1982; Campbell et al., 1985). A informação sobre a avaliação da maturidade pulmonar fetal pela relação lecitina/esfingomielina no líquido amniótico, utilizada em muitos estudos como critério de exclusão, mas raramente realizada pelas maternidades locais, não estava disponível no banco de dados analisado.

Procurou-se avaliar a validade e consistência do diagnóstico trabalho de parto prematuro, especialmente com relação aos diagnósticos diferenciais pródromos de trabalho de parto e falso trabalho de parto. O diagnóstico de trabalho de parto prematuro foi validado na referida HCP sempre que possível pelas informações relativas à dilatação e à idade gestacional, 
não estando disponível a informação sobre a contratilidade uterina. A edição e correção de dados obstétricos são descritas com maior detalhe em outro estudo (Krauss-Silva et al., 1998).

Ambos os campos relativos às exclusões existentes no banco de dados - diagnósticos de pré-natal e diagnósticos de alta hospitalar - foram utilizados para a identificação de pacientes a serem excluídas. As patologias excluídas apresentaram no banco utilizado para o presente estudo incidências geralmente semelhantes àquelas esperadas segundo a literatura (Brundenell, 1984; Dunlop et al., 1986; Amon et al., 1988; Ginsberg-Feller, 1988; Wenstrom, 1992; Sullivan \& Ramanathan, 1994; Zuspan \& Quilligan, 1994; Ferguson II \& Besinger, 1996).

Quanto ao procedimento corticoterapia, o sumário de alta previa apenas registro sobre a sua utilização ou não, não compreendendo dados sobre o momento do início do tratamento nem sobre sua completude.

A edição e correção de dados neonatais foi realizada para idade gestacional, para o diagnóstico de SAR e para morte neonatal. Também foi editada e corrigida a união dos arquivos das mães e filhos relativos ao presente estudo, feita por intermédio do número da HCP comum ao conjunto mãe-filho.

A avaliação da idade gestacional do recémnascido pelo método de Dubowitz - efetuada por alguns dos ensaios; - não estava disponível; foi estimada localmente pelo método de Capurro (somático), apresentando registro bastante satisfatório.

Considerando-se que, para diagnosticar a síndrome de angústia respiratória, utilizam-se parâmetros relativamente bem definidos e consensuais entre os neonatologistas, analisamos a freqüência de SAR e de seu diagnóstico diferencial principal - a taquipnéia transitória que apresentaram incidências de 1,7\% e 2,9\%, respectivamente, compatíveis com a literatura (Kopelman, 1984; Wald, 1984). Por outro lado, os diagnósticos de hemorragia intraventricular e enterocolite necrotizante estavam sub-registrados, provavelmente, devido a serem insuficientes os meios diagnósticos nos serviços estudados.

Referentes a serem utilizados

pelo presente estudo

O presente estudo priorizou faixas de idade gestacional para avaliação tendo em vista a eficácia da corticoterapia e a viabilidade do recém-nascido com os recursos da atenção neonatal local. Foi focalizada a faixa de 28 semanas a 33 semanas e seis dias e secundariamen- te a faixa de 28 semanas a 36 semanas e seis dias. Para examinar o benefício relativo a diferentes faixas de idade gestacional, isto é, a população de potenciais beneficiários da corticoterapia segundo faixa de idade gestacional em uma dada população, levaram-se em conta não apenas o percentual de recém-nascidos vivos, a incidência de condições mórbidas, e a mortalidade por intervalo de idade gestacional mas também a eficácia e o custo-efetividade da assistência neonatal relacionadas a esses intervalos.

Para a caracterização da fase do trabalho de parto prematuro que conformou o referente desse estudo, utilizamos um critério assumido pela maioria dos ensaios clínicos de drogas tocolíticas (Krauss-Silva et al., 1999) - que propiciam a interrupção provisória do trabalho de parto, permitindo a ação da corticoterapia - dilatação cervical à admissão hospitalar entre $2 \mathrm{e}$ $4 \mathrm{~cm}$.

Os referentes criados com base nesta metodologia foram:

a) Gestantes com idade gestacional entre 28 semanas e 33 semanas e seis dias, em trabalho de parto prematuro, com dilatação de 2 a $4 \mathrm{~cm}$ inclusive, excluindo pacientes com contra-indicação médica ao uso de corticóides: sinais de infecção intra-uterina e/ou amnionite, morte fetal, eclâmpsia, sofrimento fetal agudo, hemorragias do terceiro trimestre, alergias ao uso de corticóides, doenças imunossupressoras (exclusões absolutas).

b) Gestantes com idade gestacional entre 28 semanas e 33 semanas e seis dias, em trabalho de parto prematuro, com dilatação de 2 a 4 $\mathrm{cm}$ inclusive, excluindo pacientes com contra-indicação médica ao uso de corticóides: sinais de infecção intra-uterina e/ou amnionite, morte fetal, eclâmpsia, sofrimento fetal, hemorragias do terceiro trimestre, alergias ao uso de corticóides, doenças imunossupressoras, DHEG (doença hipertensiva específica da gravidez) grave, diabetes, crescimento intrauterino retardado e isoimunização ABO e Rh (exclusões absolutas + relativas).

c) Idem ao referente b, exceto quanto à faixa de idade gestacional: 28 a 36 semanas e seis dias.

\section{Indicadores e padrões de processo}

Considerando-se as limitações já mencionadas da fonte de dados, a análise do processo da assistência mediu apenas o percentual de realização de corticoterapia antenatal. O padrão de processo para os referentes delimitados foi de $100 \%$. 


\section{Indicadores e padrões de resultado}

Para estimar os padrões de resultado para esse estudo - incidência esperada de SAR e mortalidade neonatal para os referentes previstos era importante que a estrutura em termos de idade gestacional ao parto dos serviços a serem avaliados fosse semelhante à estrutura das pacientes incluídas nos estudos controlados dos quais seriam derivados os padrões, uma vez que a incidência de SAR e a mortalidade neonatal variam grandemente e a eficácia da corticoterapia antenatal também varia com a idade gestacional. Alternativamente, era importante que os resultados dos ensaios estivessem disponíveis segundo intervalos de idade gestacional suficientemente pequenos para o propósito em pauta, já que não dispomos do banco de dados desses ensaios.

Para essa estimativa, procuramos primeiramente avaliar as conseqüências sobre tais resultados dos três principais métodos de recorte da idade gestacional efetuados pelos ensaios: utilização exclusiva da DUM/USG, utilização da DUM/USG e complementarmente o critério da relação L/E superior a 2 (aproximadamente) e utilização da DUM/USG e, complementarmente, o critério da idade gestacional do bebê, segundo Dubowitz, maior que 34 semanas.

O efeito da exclusão pelo critério L/E torna inválida a comparação de resultados entre os ensaios e destes com os serviços locais (sem disponibilidade de dosagem de L/E), já que esse efeito (critério L/E) - exclusão das pacientes com baixo risco de imaturidade pulmonar - altera substancial e diferencialmente as chances originais de ocorrência de SAR e de mortalidade correspondente a cada intervalo de idade gestacional (Wald, 1984). Quanto aos estudos que excluíram pacientes por meio do critério Dubowitz > 34 semanas, além da relativamente pequena modificação da estrutura de idade gestacional efetuada pela classificação de Dubowitz em si, esses trabalhos não apresentavam as informações sobre a estrutura de idade gestacional necessárias à avaliação em pauta (faixas de idade gestacional e estratificação segundo idade gestacional à admissão e ao parto, média e desvio padrão da idade gestacional à admissão/parto e média de tempo decorrido entre a admissão e a alta para o grupo tratado e controle). Também não apresentavam resultados segundo intervalos de idade gestacional.

Finalmente, comparamos a estrutura de idade gestacional das pacientes dos estudos com idade gestacional estimada exclusivamente por USG/DUM (Liggins \& Howie, 1972; Gam- su et al., 1989), métodos também utilizados nos serviços estudados, à estrutura correspondente às pacientes desses serviços. A estrutura do estudo de Liggins \& Howie (1972), o maior dos ensaios, era bastante diferente daquela dos serviços, invalidando a utilização do resultado desse estudo como padrão. Não foi possível a comparação da estrutura de idade gestacional do estudo de Gamsu et al. (1989) com a do nosso banco, pois esse estudo não informa suficientemente sobre a estrutura de idade gestacional das pacientes (Tabela 3). Por outro lado, esses estudos também não informavam os resultados segundo intervalos de idade gestacional suficientemente pequenos para permitir um eventual ajuste do padrão quanto à interveniência dessa variável. Dessa maneira, não conseguimos realizar o adequado controle da idade gestacional, o que permitiria estimar padrões de resultado - incidência esperada de SAR e mortalidade neonatal para os referentes previstos - para esta avaliação.

\section{Resultados: avaliação da qualidade}

\section{Análise de processo}

A realização da corticoterapia nos serviços estudados para o referente trabalho de parto prematuro na faixa de idade gestacional de 28 a 33 semanas e seis dias, excluídas as contra-indicações absolutas já referidas, foi irrisória, 4,3\% (Tabela 4). Considerando-se, para o mesmo referente, as contra-indicações absolutas e relativas, a realização da corticoterapia antenatal permaneceu muito pequena: $4,4 \%$ (Tabela 5 ). Para a faixa de idade gestacional de 28 semanas a 36 semanas e seis dias, a utilização de corticoterapia antenatal foi ainda menor: $2,3 \%$.

Analisando-se o uso de corticoterapia no reduzido subgrupo de pacientes que fizeram uso de tocolíticos (18,7\% do total de pacientes na faixa de idade gestacional de 28 a 36 semanas e seis dias, e, $10,5 \%$ do total de pacientes na faixa de idade gestacional de 28 a 33 semanas e seis dias), o resultado foi um percentual um pouco mais alto - $11,5 \%$, na faixa de 28 a 33 semanas e seis dias e $8,9 \%$, na faixa de 28 a 36 semanas e seis dias (Tabela 6).

\section{Análise de resultados}

Não foi possível realizar a análise de resultados, uma vez que não conseguimos estimar padrões para os referentes previstos. 
Tabela 3

Idade gestacional e incidência de RDS para os trabalhos que selecionaram a idade gestacional pela USG e DUM.

\begin{tabular}{|c|c|c|c|c|}
\hline Ensaios & $\begin{array}{l}\text { Liggins \& Howie } \\
\text { (1972) (parcial) }\end{array}$ & $\begin{array}{l}\text { Gamsu et al. } \\
\text { (1989) }\end{array}$ & $\begin{array}{l}\text { Garite et al. } \\
\text { (1992) }\end{array}$ & $\begin{array}{l}\text { Schutte et al. } \\
(1980)^{\star \star}\end{array}$ \\
\hline Faixa da idade à admissão & $24-36,6^{*}$ & $25-33,6$ & $24-28(27$ e $6 d)$ & $26<32$ \\
\hline $\begin{array}{l}\text { Média da idade gestacional à admissão } \\
\text { grupo controle/desvio padrão }\end{array}$ & $C: 32,14 / 4,2$ semanas & $C: 31,4 / 2,4$ & $C: 25,8 / 1,3$ & $C: N D$ \\
\hline $\begin{array}{l}\text { Média da idade gestacional à admissão } \\
\text { grupo tratado/desvio padrão }\end{array}$ & $\begin{array}{l}\mathrm{T}: 31,71 / 3 \\
\text { semanas (21 dias) }\end{array}$ & $\mathrm{T}: 31,5 / 2,5$ & $\mathrm{~T}: 25,5 / 1,2$ & $\mathrm{~T}: \mathrm{ND}$ \\
\hline $\begin{array}{l}\text { Média da idade gestacional ao nascimento } \\
\text { Grupo controle/ desvio padrão }\end{array}$ & $\begin{array}{l}\text { (DUM) } \\
34,85 / 2,6 \text { semanas }\end{array}$ & $\begin{array}{l}\text { (DUM) } \\
33,5 / 4,2 \text { semanas }\end{array}$ & ND & ND \\
\hline $\begin{array}{l}\text { Média da idade gestacional ao nascimento } \\
\text { Grupo tratado/ desvio padrão }\end{array}$ & $\begin{array}{l}\text { (DUM) } \\
35,57 / 4 \text { semanas } 4 \text { dias }\end{array}$ & $\begin{array}{l}\text { (DUM) } \\
34,6 / 3,8 \text { semanas }\end{array}$ & ND & ND \\
\hline Delta tempo grupo controle/desvio padrão & $\begin{array}{l}2 \text { semanas } 4 \text { dias } \\
(14,4 \text { dias })\end{array}$ & 2,2 semanas & $\begin{array}{l}\text { Dias/Pacientes } \\
0-1: 11 \\
2-7: 17 \\
>8: 12\end{array}$ & ND \\
\hline Delta tempo grupo tratado/ desvio padrão & $\begin{array}{l}3 \text { semanas } 2 \text { dias } \\
(22,1 \text { dias })\end{array}$ & 3,1 semanas & $\begin{array}{l}\text { Dias/Pacientes } \\
0-1: 6 \\
2-7: 13 \\
>8: 14\end{array}$ & ND \\
\hline RDS/ grupo Tratado & $11 / 122$ & $7 / 131$ & $21 / 33$ & $11 / 49$ \\
\hline RDS/grupo controle & $25 / 97$ & $16 / 137$ & $28 / 40$ & $17 / 46$ \\
\hline $\begin{array}{l}\text { Incidência de RDS do grupo tratado } \\
\text { e grupo controle }\end{array}$ & $\begin{array}{l}\text { Trat: } 9,01 \\
\text { Cont: } 25,77\end{array}$ & $\begin{array}{l}\text { Trat: } 5,34 \\
\text { Cont: } 11,67\end{array}$ & $\begin{array}{l}\text { Trat: } 63,63 \\
\text { Cont: } 70,00\end{array}$ & $\begin{array}{l}\text { Trat: } 22,44 \\
\text { Cont: } 36,95\end{array}$ \\
\hline $\begin{array}{l}\text { Incidência de RDS do grupo controle } \\
\text { na faixa de 28-34 semanas }\end{array}$ & ND & ND & ND & ND \\
\hline
\end{tabular}

* A estrutura do Liggins \& Howie (1972) tem faixa abaixo de 32 semanas relativamente mais representada que o esperado, assim como a faixa acima de 34 semanas.

$\star *$ Esses resultados referem-se às pacientes que pariram antes de completar 34 semanas de gestação:

foram excluídas 25 pacientes ou $20,83 \%$.

Tabela 4

Realização de corticoterapia antenatal em pacientes em trabalho de parto prematuro, considerando-se as exclusões absolutas, em maternidades públicas do Rio de Janeiro.

\begin{tabular}{|c|c|c|c|c|c|c|}
\hline \multirow[t]{3}{*}{ Corticoterapia } & \multicolumn{6}{|c|}{ Faixas de Idade Gestacional (DUM/USG) } \\
\hline & \multicolumn{2}{|c|}{$<28$ semanas } & \multicolumn{2}{|c|}{28 a 33 semanas e seis dias } & \multicolumn{2}{|c|}{34 a 36 semanas e seis dias } \\
\hline & $\mathrm{n}$ & $\%$ & $\mathrm{n}$ & $\%$ & $\mathrm{n}$ & $\%$ \\
\hline $\operatorname{Sim}$ & 1 & 1,6 & 13 & 4,3 & 7 & 1,3 \\
\hline Não & 60 & 98,4 & 288 & 95,7 & 548 & 98,7 \\
\hline Total & 61 & 100 & 301 & 100 & 555 & 100 \\
\hline
\end{tabular}




\section{Discussão}

Embora tenhamos baseado esse trabalho na revisão sistemática de Crowley (1996) - atualizada e de boa qualidade (Clarke \& Stewart, 1984; Chalmers et al., 1987; Chalmers \& Lau, 1993), a maioria dos estudos que comprovaram os benefícios da corticoterapia antenatal foram realizados na década de 70 e início de 80 , conforme citações feitas anteriormente.

A despeito de não ser possível estimar padrões de resultado e compará-los com os resultados observados nos serviços avaliados, a evidência científica existente permite deduzir que os resultados obtidos pelos serviços foram com certeza piores que aqueles que seriam observados caso a corticoterapia houvesse sido empregada pelos serviços nos casos para os quais está indicada.

A tentativa feita nesse trabalho de utilizar resultados obtidos - no grupo tratado - em ensaios clínicos controlados como estimativa do padrão de resultado, tem como argumentos: a) a simplicidade da técnica envolvida na execução do procedimento corticoterapia antenatal e do diagnóstico associado, trabalho de parto prematuro e b) o grande número de ensaios envolvidos e a variedade de critérios de inclusão e exclusão neles utilizados.

Os ensaios analisados não definem os critérios de inclusão com precisão, principalmente em relação ao diagnóstico trabalho de parto prematuro, referido simplesmente como trabalho de parto prematuro ou trabalho de parto prematuro não planejado, sem menção a critérios de contratilidade uterina ou dilatação cervical. Por isso, utilizamos o critério dilatação cervical 2 a $4 \mathrm{~cm}$, geralmente assumido pelos ensaios de tocolíticos (Krauss-Silva et al.,1998).

As condições de exclusão diabetes e amnionite, incorporadas ao referente do presente estudo, encontraram-se sub-registradas no banco de dados utilizado (Dunlop et al., 1986; Amon et al., 1988; Ginsberg-Feller, 1988; Zuspan \& Quilligan, 1994). Os percentuais sub-registrados constituíram, todavia, frações muito pequenas do referente do presente trabalho, não podendo influenciar, por isso, de forma relevante, os resultados obtidos.

Os ensaios com $85 \%$ de pacientes em trabalho de parto (Papageorgiou et al., 1979; Doran et al., 1980; Gamsu et al., 1989) apresentaram melhor resultado (RT: $0,36(0,24<\mathrm{RT}<0,54))$ que o resultado geral para o conjunto de ensaios (RT: 0,64 $(0,57<\mathrm{RT}<0,75))$. Entretanto, a estrutura de idade gestacional das pacientes desses ensaios era claramente mais favorável à ação da corticoterapia antenatal (com média de cer-
Tabela 5

Realização de corticoterapia antenatal em pacientes em trabalho de parto prematuro, considerando-se as exclusões absolutas e relativas, em maternidades públicas do Rio de Janeiro.

\begin{tabular}{lcccc}
\hline Corticoterapia & \multicolumn{4}{c}{ Faixas de Idade Gestacional (DUM/USG) } \\
& 28 a 33 semanas e seis dias & 28 a & 36 & semanas e seis dias \\
& $n$ & $\%$ & $n$ & $\%$ \\
\hline Sim & 13 & 4,4 & 20 & 2,3 \\
Não & 286 & 95,6 & 827 & 97,7 \\
Total & 299 & 100 & 847 & 100 \\
\hline
\end{tabular}

Tabela 6

Realização da corticoterapia antenatal em pacientes em trabalho de parto prematuro que utilizaram tocolíticos, considerando-se as exclusões absolutas, em maternidades públicas do Rio de Janeiro.

\begin{tabular}{|c|c|c|c|c|}
\hline \multirow[t]{3}{*}{ Corticoterapia } & \multicolumn{4}{|c|}{ Faixas de Idade Gestacional (DUM/USG) } \\
\hline & \multicolumn{2}{|c|}{28 a 33 semanas e seis dias } & \multicolumn{2}{|c|}{28 a 36 semanas e seis dias } \\
\hline & $\mathrm{n}$ & $\%$ & $n$ & $\%$ \\
\hline Sim & 11 & 11,5 & 14 & 8,9 \\
\hline Não & 85 & 88,5 & 144 & 91,1 \\
\hline Total & 96 & 100 & 158 & 100 \\
\hline
\end{tabular}

ca de 33 semanas ao parto), que a estrutura do conjunto dos ensaios, que incluiu muitas pacientes em intervalos de idade gestacional desfavorável (menor que 30 semanas e maior que 34 semanas), não sendo possível, por isso, inferir diferença de eficácia.

A incidência da síndrome de angústia respiratória do recém-nascido é três vezes maior na faixa de idade gestacional entre 28 e 33 semanas e seis dias (37\%) do que na faixa entre 34 e 35 semanas (12\%) e sua letalidade é sete vezes maior naquela faixa $(44 \%)$ do que nessa (6\%). Na faixa menor que 28 semanas, a incidência e a letalidade da SAR é quase duas vezes maior que na faixa de 28 a 33 semanas e seis dias (Wald, 1984). Todavia, a letalidade por SAR, assim como a mortalidade neonatal em geral, varia de acordo com os recursos e o desempenho dos serviços (Robertson et al., 1992). Por outro lado, o número de bebês pré-termo nascidos varia diretamente com a idade gestacional. Computando-se esses fatores e ainda os dados sobre eficácia e sobre custo-efetividade da assistência neonatal de diferentes níveis (Krauss-Silva, 1992), conclui-se que a faixa de 
28 semanas e 33 semanas e seis dias contém um maior número e percentual de beneficiários potenciais da corticoterapia que a faixa seguinte e a anterior tanto em casos quanto em mortes por SAR, tendo ainda uma relação de custo-efetividade muito mais favorável que a faixa de idade gestacional anterior. A inclusão nos referentes do presente trabalho das gestações com duração entre 28 e 30 semanas, intervalo em que a eficácia da corticoterapia antenatal é relativamente pequena, levou em conta a alta mortalidade de recém-natos nessa faixa de idade gestacional, mesmo com a utilização de recursos neonatais complexos.

Conforme relatado na Metodologia, as tentativas de elaborar padrão de resultado válido para os referentes desse trabalho foram frustradas: a) porque vários dos ensaios tinham número de participantes pequeno e não trabalharam com indicadores de mortalidade, e sim de morbidade (SAR, HIV etc); b) pela escassez de informação relativa à distribuição da idade gestacional e resultados associados a diferentes faixas de idade gestacional, importante fator de risco para morte neonatal e SAR e modificador do efeito da corticoterapia antenatal, b) pelas intervenções realizadas pelos diferentes protocolos na definição da população do estudo, modificando o critério idade gestacional à admissão, e c) pela dificuldade de acesso ao banco de dados dos ensaios (Miettinen, 1985; Horwitz, 1987; Yusuf et al., 1991; GAO/EUA, 1992; Oxman \& Guyatt, 1992; Eysenck, 1995; Rothman \& Greenland, 1998). Caso a questão da interveniência da idade gestacional no resultado morte neonatal houvesse sido resolvida, outro problema, todavia, deveria ser enfrentado quanto à estimativa do padrão: o diferencial de efetividade da assistência neonatal entre os serviços estudados e os serviços correspondentes aos ensaios.

O baixo uso de corticoterapia observado nos serviços foi consistente com o pequeno percentual de uso de tocolíticos para aquelas pacientes, percentual este consistente com o baixíssimo percentual de trabalhos de parto revertidos (Krauss-Silva et al., 1998). Os resultados da cuidadosa edição dos dados analisados indicam boa qualidade no preenchimento de dados em geral. Os resultados do referido trabalho relativo ao uso de tocolíticos falam a favor, por outro lado, de sub-registro de trabalho de parto prematuro, possivelmente por erro diagnóstico (falso trabalho), o que poderia piorar o resultado observado quanto à utilização da corticoterapia.

O baixo uso dos tocolíticos betamiméticos e da corticoterapia antenatal parece ser em parte relacionado à falta de informação sobre a eficácia e segurança dessas drogas na prevenção de danos neonatais relacionados ao parto prematuro. Utilização muito baixa da corticoterapia foi também observada no início da década de 90 nos Estados Unidos - em contraste com o que ocorria na Europa, motivando a realização pelo governo americano de um consenso sobre o assunto (NICH, 1994). Embora os dados do presente estudo também se refiram ao início da década de 90, baixo uso dessas tecnologias foi observado para o ano de $1995 \mathrm{em}$ maternidade municipal terciária do Rio de Janeiro (Albuquerque, 1998).

Deixou-se de avaliar, nesse trabalho, parte importante do referente da corticoterapia antenatal: a parte dos partos prematuros eletivos, que compreende cerca de $25 \%$ dos nascimentos prematuros. A preocupação em particular com a fração não-eletiva das indicações da corticoterapia antenatal, correspondente a mais de $50 \%$ dos partos prematuros - o percentual restante relacionado à morte anteparto e malformações letais (Chalmers et al., 1993) - devese ao caráter de emergência desse tipo de atendimento, que implica elevado grau de prontidão dos profissionais de saúde, dada a necessidade de tocólise para o uso da corticoterapia, o que também pode concorrer para o baixo uso do conjunto tocolítico-corticoterapia.

Além de ser consideravelmente eficaz na prevenção de diversas condições mórbidas e morte neonatal associadas com nascimento prematuro, a corticoterapia antenatal tem muito baixo custo quando comparada às alternativas existentes. Os custos associados ao tratamento completo com a droga, o que implica a aplicação de duas injeções intramusculares, são inferiores a 50 reais. Considerando-se que essa tecnologia, no caso do trabalho de parto prematuro, deve ser complementada por uma infusão endovenosa de tocolíticos, substâncias inibidoras do trabalho de parto, os custos associados à intervenção preventiva completa são de aproximadamente 100 reais, mais os custos de cerca de dois dias dias extras de internação obstétrica, por paciente. Por outro lado, as alternativas neonatais mais tradicionais para tratamento de condições mórbidas de recém-nascidos prematuros, mais ou menos graves, são relacionadas a tecnologias de nível secundário e terciário, sendo dezenas ou centenas de vezes mais caras que a corticoterapia antenatal (Krauss-Silva, 1992). Com relação às tecnologias mais recentes, o custo médio de aquisição do surfactante pulmonar exógeno, tecnologia alternativa ou complementar à corticoterapia antenatal, crescentemente utilizada para subs- 
tituir o surfactante natural e prevenir/tratar doenças respiratórias (principalmente a SAR) em recém-natos prematuros, é de cerca de 1800 reais. O custo desse procedimento deve ainda incluir o uso de recursos humanos e materiais como sala de parto e berçário terciário, compreendendo equipe médica e de enfermagem treinada, monitorização contínua e utilização de diversos equipamentos e procedimentos complementares. Portanto, o custo associado à realização do procedimento surfactante pulmonar é muito alto, chegando em média a vários milhares de reais, especialmente tendo em conta que sua eficácia sobre a mortalidade neonatal é menos relevante que a da corticoterapia antenatal e que sua efetividade é provavelmente baixa em UTIs neonatais brasileiras.

\section{Agradecimentos}

Esse trabalho recebeu auxílio financeiro da Organização Panamericana de Saúde (HDP/HDR(DRC/RG/ BRA/93-956)), Fundação Oswaldo Cruz (PAPES no 109), Fundação de Amparo à Pesquisa do Rio de Janeiro e Conselho Nacional de Desenvolvimento Científico e Tecnológico.

\section{Referências}

ALBUQUERQUE, C. P., 1998. Avaliação da Qualidade da Assistência Perinatal ao Parto Prematuro. Relatório de pesquisa. Rio de Janeiro: Fundação de Amparo à Pesquisa do Estado do Rio de Janeiro FAPERJ.(mimeo.)

AMON, E.; LEWIS, S. V.; SIBAI, B. M.; VILLAR, M. A. \& ARHEART, K. L., 1988. Ampicillin prophylaxis in preterm premature rupture of the membranes: A prospective randomized study. American Journal of Obstetrics and Gynecology, 159:539-543.

ANDERSON, R. S.; PHILLIPS, P. J. \& MIDWINTER, A. 1982. Routine real-time scanning at the first hospital visit. British Journal of Obstetrics and Gynaecology, 89:16-19.

BAILEY, K. R., 1987. Inter-study differences: How should they influence the interpretation and analysis of results? Statistics in Medicine, 6:359360.

BANTA, H. D. \& LUCE, B. R., 1993. Health Care Technology and its Assessment. London: Oxford University Press.

BRUNDENELL, M., 1984. Diabetes. In: Medical and Surgical Problems in Obstetrics (M. Brundenell \& P. L. Wilds, eds.), pp. 120-40, Bristol: John Wright \& Sons.

\section{Conclusão}

O estudo revelou um uso muito reduzido de corticoterapia antenatal no trabalho de parto prematuro para o subgrupo de idade gestacional entre 28 e 36 semanas e seis dias e também para o subgrupo de maior benefício, ou seja, entre 28 e 34 semanas, excluídas as contra-indicações, o que indica má qualidade da assistência hospitalar a essa condição em maternidades públicas do Rio de Janeiro de níveis secundário e terciário. Essa conclusão merece a atenção dos profissionais que atuam na assistência e também dos planejadores e gestores do setor, tendo em vista os benefícios e os custos dessa tecnologia em comparação com aqueles correspondentes às alternativas existentes na área neonatal, e também o fato de que as alternativas neonatais são tecnologicamente mais complexas e, portanto, de incorporação mais difícil.
BLOCK, M. F.; KING, O. R. \& CRESBY, W. M., 1977. Antenatal glucocorticoid therapy for the prevention of respiratory distress syndrome in the premature infant. Obstetrics and Gynecology, 50:186-190.

CAMPBELL, S.; WASOF, S. L.; LITTLE, D. \& COOPER, D. J., 1985. Routine ultrasound screening for the prediction of gestational age. Obstetrics and $G y$ necology, 65:613-620.

CARARACH, V.; SENTIS, J.; BOTET, F. \& DE LOS RIOS, L., 1990. A multicentric prospective randomized study in premature rupture of membranes (PROM). Respiratory and infectious complications in the newborn. Proceedings of 12th European Congress of Perinatal Medicine, p. 216. Available in: Pregnancy and Childbirth Module of The Cochrane Database of Systematic Reviews (J. P. Neilson, C. A. Crowther, E. D. Hodnett, G. J. Hofmeyr \& M. J. N. C. Keirse, eds.). The Cochrane Library [database on disk and CDROM], Disk Issue 2. 1997. Oxford: The Cochrane Collaboration, Update Software.

CARLAN, S. J.; PARSONS, M.; O'BRIEN, W. F. \& KRAMMER, J., 1991. Pharmacologic pulmonary maturation in preterm premature rupture of membranes. American Journal of Obstetrics and Gynecology, 164:371-375. 
CHALMERS, T. C.; LEVIN, H.; SACKS, H. S.; REITMAN, R.; BERRIER, J. \& NAGALINGAM, R., 1987. Metaanalysis of clinical trials as a scientific discipline. I: Control of bias and comparison with large cooperative trials. Statistics in Medicine, 6:315-325.

CHALMERS, T. C. \& LAU, J., 1993. Meta-analytic stimulus for changes in clinical trials. Statistical Methods in Medical Research, 2:161-172.

CHALMERS, I.; ENKIN, M. \& KEIRSE, M. J. N. C., 1993. Effective Care in Pregnancy and Chilbirth. New York: Oxford Medical Publications.

CHALMERS, I. \& ALTMAN, D. G., 1995. Systematic Reviews. London: BMJ Publishing Group - BMA House.

CLARKE, M. J. \& STEWART, L., 1994. Systematic Reviews - Obtaining data from randomised controlled trials: How much do we need for reliable and informative meta-analyses? British Medical Journal, 309:1007-1010.

COLLABORATIVE GROUP ON ANTENATAL STEROID THERAPY [US Antenatal Steroid Trial], 1981. Effect of antenatal dexamethasone administration on the prevention of respiratory distress syndrome. American Journal of Obstetrics and Gynecology, 141:276-287.

CROWLEY, P.; CHALMERS, I. \& KEIRSE, M. J. N. C., 1990. The effects of corticosteroid administration before preterm delivery: A review of the evidence from controlled trials. British Journal of Obstetrics and Gynaecology, 97:11-25.

CROWLEY, P., 1996. Corticosteroids prior to preterm delivery. In: Pregnancy and Childbirth Module of The Cochrane Database of Systematic Reviews (J. P. Neilson, C. A. Crowther, E. D. Hodnett, G. J. Hofmeyr \& M. J. N. C. Keirse, eds.), available in The Cochrane Library [database on disk and CDROM], Disk Issue 2. Oxford: The Cochrane Collaboration, Update Software.

DORAN, T. A.; SWYER, P.; MacMURRAY, B.; MAHON, W.; ENHOMING, G.; BEMSTEIN, A.; FALK, M. \& WOOD, M. M., 1980. Results of a double blind controlled study on the use of betamethasone in the prevention of respiratory distress syndrome. American Journal of Obstetrics and Gynecology, 136:313-320.

DUNLOP, P. D. M.; CROWLEY, P. A.; LAMONT, R. F. \& HAWKINS, D. F., 1986. Preterm ruptured membranes, no contractions. Journal of Obstetrics and Gynecology, 7:92-96.

EYSENCK, H. J., 1995. Problems with meta-analysis. In: Systematic Reviews (I. Chalmers \& D. G. Altman, eds.), pp. 64-74, London: BMJ Publishing Group.

FERGUSON II, J. E. \& BESINGER, R. E., 1996. Preterm labor. In: Intrapartum Obstetrics (J. T. Repke, ed.), pp. 275-295, New York: Churchill Livingstone.

GAMSU, H. R.; MULLINGER, B. M.; DONNAI, P. \& DASH, C. H., 1989. Antenatal administration of betamethasone to prevent respiratory distress syndrome in preterm infants: Report of a UK multicentre trial. British Journal of Obstetrics and Gynaecology, 96:401-410.

GARITE, T. J.; RUMNEY, P. J.; BRIGGS, G. G.; HARDING, J. A.; NAGEOTTE, M. P.; TOWERS, C. V. \& FREEMAN, R. K., 1992. A randomized placebocontrolled trial of betamethasone for the preven- tion of respiratory distress syndrome at 24 to 28 weeks' gestation. American Journal of Obstetrics and Gynecology, 166:646-651.

GINSBERG-FELLER, F., 1988. Epidemiology, genetics and imunology. In: Diabetes Mellitus in Pregnancy: Principles and Pratice (A. Reece \& D. R. Coulsan, eds.), pp. 17-45, New York: Churchill Livingstone Inc.

HORWITZ, R. I., 1987. Complexity and contradiction in clinical trial research. American Journal of Medicine, 82:498-510.

GAO/EUA (United States General Accounting Office/ Program Evaluation and Methodology Division), 1992. Cross Design Synthesis: A New Strategy for Medical Effectiveness Research. Report to Congressional Requesters. Document GAO/PEMD92-18, Washington, D.C.: GAO/PEMD.

KARI, A. M.; HALLMAN, M.; ERONEN, M.; TERAMO, K.; VITANEN, M.; KOIVISTO, M. \& IKONEN, R. S., 1994. Prenatal dexamethasone in conjunction with rescue therapy of human surfactant - A randomized placebo controlled multicenter study. Pediatrics, 93:730-736.

KOPELMAN, B. I., 1984. Distúrbios Respiratórios do Recém-nascido. São Paulo: Livraria Atheneu.

KRAUSS-SILVA, L., 1992. Technology Assessment of Different Levels of Neonatal Care. Ph.D. Thesis, Birmingham: Health Services Management Centre, University of Birmingham.

KRAUSS-SILVA, L., 1996. Perinatal information system: A methodological proposal. International Journal of Quality in Health Care, 8:279-289.

KRAUSS-SILVA, L.; REIS, A. F.; COSTA, T. P.; ALBUQUERQUE, C. P.; AZEVEDO, A. P. \& IAMADA, N. O. I., 1999. Análise da adequação e efetividade do uso de tocolíticos no trabalho de parto prematuro. Cadernos de Saúde Pública, 15:581-590.

LIGGINS, G. C. \& HOWIE, R. N., 1972. A controlled trial of antepartum glucocorticoid treatment for prevention of the respiratory distress syndrome in premature infants. Pediatrics, 50:515525.

LOCKWOOD, C. J., 1994. Recent advances in elucidating the pathogenesis of preterm delivery, the detection of patients at risk, and preventative therapies. Obstetrics and Gynecology, 6:7-18.

MIETTINEN, O. S., 1985. Theoretical Epidemiology. New York: John Wiley \& Sons.

MORALES, W. J.; DIEBEL, N. D.; LAZAR, A. J. \& ZADROZNY, D., 1986. The effect of antenatal dexamethasone administration on the prevention of respiratory distress syndrome in preterm gestations with premature rupture of membranes. American Journal of Obstetrics and Gynecology, 154:591-595.

MORRISON, J. C.; WHYBREW, W. D.; BUCOVAZ, E. T. \& SCHNEIDER, J. M., 1978. Injection of corticosteroids into mother to prevent neonatal respiratory distress syndrome. American Journal of $\mathrm{Ob}$ stetrics and Gynecology, 131:358-366.

NICH/USA (National Institute of Child Health and Human Development/United States of America), 1994. Report of the Consensus Development Conference on the Effect of Corticosteroids for Fetal Maturation on Perinatal Outcomes. NIH Publication No. 95-3784. Bethesda: NICH. 
OTA/USA (Office of Technology Assessment/United States of America), 1994. Identifying Health Technologies that Work. Washington, DC: U.S. Goverment Printing Office.

OXMAN, A. D. \& GUYATT, G. H., 1992. A consumer's guide to subgroup analyses. Annals of Internal Medicine, 116:78-84.

OXMAN, A. D.; COOK, D. J. \& GUYATT, G. H., 1994. User's guide to the medical literature. VI - How to use an overview. JAMA, 272:1367-1371.

PAPAGEORGIOU, A. N.; DESGRANGES, M. F.; MASSON, M.; COLLE, E.; SHATZ, R. \& GELFAND, M. M., 1979. The antenatal use of betamethasone in the prevention of respiratory distress syndrome: A controlled double-blind study. Pediatrics, 63: 73-79.

PARSONS, M. T.; SOBEL, D.; CUMMISKEY, K.; CONSTANTINE, L. \& ROITMAN, J., 1988. Steroid, antibiotic and tocolytic $v s$ no steroid antibiotic and tocolytic management in patients with preterm PROM at 25-32 weeks. In: Proceedings of 8th Annual Meeting of the Society of Perinatal Obstetricians, p. 44. Available in: Pregnancy and Childbirth Module of The Cochrane Database of Systematic Reviews (J. P. Neilson, C. A. Crowther, E. D. Hodnett, G. J. Hofmeyr \& M. J. N. C. Keirse, eds.). The Cochrane Library [database on disk and CDROM], Disk Issue 2. 1997. Oxford: The Cochrane Collaboration, Update Software.

ROBERTSON, P. A.; SNIDERMAN, M. D.; LAROS Jr., R. K.; COWAN, R.; HEILBRON, D.; GOLDENBERG, R. L.; IAMS, J. D. \& CREASY, K., 1992. Neonatal morbidity according to gestational age and birth weight from five tertiary care centers in the United States, 1983 through 1986. American Journal of Obstetrics and Gynecology, 166:1629-1645.

ROPER, W. L.; WINKENWERDER, W.; HACKBARTH, G. M. \& KRAKAUER, H., 1988. Effectiveness in health care: An initiative to evaluate and improve medical practice. New England Journal of Medicine, 319:1197-1202.

ROTHMAN, K. J. \& GREENLAND, S., 1998. Modern Epidemiology. 2nd. Ed. Philadelphia: LippincottRaven.

SACKES, H. S.; BERRIER, J.; REITMAN, D.; BERK, A. \& CHALMERS, T. C., 1987. Meta-analyses of randomized controlled trials. New England Journal of Medicine, 316:450-455.

SCHMIDT, P. L.; SIMS, M. E.; STRASSNER, H. T.; PAUL, R. H.; MUELLER, E. \& McCART, D., 1984. Effect of antepartum glucocorticoid administration upon neonatal respiratory distress syndrome and perinatal infection. American Journal of $\mathrm{Ob}$ stetrics and Gynecology, 148:178-86.

SCHUTTE, M. F.; TREFFERS, P. E.; KOPPE, J. G \& BREUR, W., 1980. The influence of betamethasone and orciprenaline on the incidence of respiratory distress syndrome in the newbom after premature labour. British Journal of Obstetrics and Gynaecology, 87:127-131.

SCHULZ, K. F.; CHALMERS, I.; HAYES, R. J. \& ALTMAN, D. G., 1995. Dimensions of methodological quality associated with estimates of treatment effects in controlled trial. JAMA, 273:408-412.

SES/RJ (Secretaria do Estado de Saúde do Rio de Janeiro/Comissão Perinatal), 1990. Avaliação da
Qualidade da Atenção Perinatal em Maternidades Públicas do Rio de Janeiro. Rio de Janeiro: SES. (mimeo.)

SILVER, R. K.; VYSKOCIL, C. R.; SOLOMON, S. L.; FARRELL, E. E.; MacGREGOR, S. N.; NEERHOF, M. G. \& RAGIN, A., 1995. Randomized trial of antenatal dexamethasone in surfactant-treated infants delivered prior to 30 weeks of gestation. American Journal of Obstetrics and Gynecology, 172:254.

SOLL, R. F., 1997. Prophylatic administration of natural surfactant extract. Available in The Cochrane Library [database on disk and CDROM], Disk Issue 2. Oxford: The Cochrane Collaboration, Update Software.

SOLL, R. F. \& MORLEY, C. J., 1997. Prophylatic surfactant $v$ s treatment with surfactant. In: Neonatal Module of the Cochrane Database of Systematic Reviews (J. C. Sinclair, M. B. Brachen, R. F. Soll, J. D. Hobar, eds.), available in The Cochrane Library [database on disk and CDROM], Disk Issue 1. Oxford: The Cochrane Collaboration, Update Software.

SULLIVAN, J. M. \& RAMANATHAN, K. B., 1984. Cardiovascular disorders. In: Medical and Surgical Problems in Obstetrics (M. Brundenell \& P. L. Wilds, eds.), pp.11-28, Bristol: John Wright \& Sons.

TAEUSCH Jr., H. W.; FRIGOLETTO, F.; KITZMILLER, J.; AVERY, M. E.; HEHRE, A.; FROMM, B.; LAWSON, E. \& NEFF, R. K., 1979. Risk of respiratory distress syndrome after prenatal dexamethasone treatment. Pediatrics, 63:64-72.

TERAMO, K.; HALLMAN, M. \& KARI, O. R., 1980. Maternal glucocorticoid in unplanned Premature labor. Controlled study on effects of betamethasone phosphate on the phospholipids of the gastric aspirate and on the adrenal cortical function of the new born infant. Pediatrics Research, 14: 326-329.

WALD, N. J., 1984. Antenatal and Neonatal Screening. Bristol: Oxford Medical Publications.

WENSTROM, K., 1992. Premature rupture of the membranes. Obstetrics and Gynecology Clinics of North America, 19:241-249.

YUSUF, S. D.; WITTES, J.; PROBSTFIELD, J. \& TYROLER, H. A., 1991. Analysis and interpretation of treatment effects in subgrups of patients in randomized clinical trials. JAMA, 266:93-98.

ZUSPAN, F. P. \& QUILLIGAN, E. J., 1994. Current Theraphy in Obstetrics and Gynecology. Philadelphia: W. B. Saunders Company. 